\title{
Case Report \\ Self-Expanding Metal Stent (SEMS) Placement to Treat Bleeding from Late Radiation Esophagitis
}

\author{
Inayat Gill ${ }_{(1)}{ }^{1}$ Bana Antonios, ${ }^{1}$ Zaid Imam ${ }^{D},{ }^{2}$ and Gehad Ghaith ${ }^{2}$ \\ ${ }^{1}$ Department of Internal Medicine, William Beaumont Hospital, Royal Oak, MI, USA \\ ${ }^{2}$ Division of Gastroenterology and Hepatology, Department of Medicine, William Beaumont Hospital, Royal Oak, MI, USA
}

Correspondence should be addressed to Zaid Imam; zaid.imam@beaumont.org

Received 18 October 2020; Revised 18 October 2020; Accepted 15 January 2021; Published 23 January 2021

Academic Editor: Yoshihiro Moriwaki

Copyright (c) 2021 Inayat Gill et al. This is an open access article distributed under the Creative Commons Attribution License, which permits unrestricted use, distribution, and reproduction in any medium, provided the original work is properly cited.

Radiation esophagitis is a serious complication occurring in patients receiving radiotherapy for head and neck cancers. Current treatment with proton pump inhibitors and mucosal protectants provides symptomatic relief with few studies showing improvement in erosive esophagitis or ulceration. Use of self-expandable metal stents (SEMS) in cases of erosive radiation esophagitis refractory to medical therapy has not been studied. We report a case of a patient presenting with recurrent hematemesis from late (chronic) radiation esophagitis with bleeding esophageal ulceration successfully treated with SEMS placement after failure of conservative medical management, proposing a possible utility for SEMS in this setting.

\section{Introduction}

Late (chronic) radiation esophagitis occurs after three months of radiation therapy completion presenting with stricture or dysmotility-related dysphagia and rarely with the feared complication of tracheoesophageal fistula or esophageal perforation [1]. Increasing edema and inflammation beyond 30 days of radiation therapy eventually leads to stricture formation as the esophagus loses its distensibility [2]. On the other hand, symptomatic acute radiation esophagitis is a rare complication occurring in $<1 \%$ of patients receiving radiation for lymphoma, lung, head, and neck cancers $[3,4]$. It typically occurs $2-3$ weeks after radiation therapy and presents with odynophagia, dysphagia, and less likely with hematemesis [4]. The mainstay treatment of radiation-related esophageal strictures is endoscopic dilation and use of proton pump inhibitor therapy [1]. The mainstay treatment for acute radiation esophagitis includes dietary modification in the form of eliminating irritants such as spicy foods and conforming to soft foods [4]. In addition, medical therapy with proton pump inhibitors (PPI), histamine blockers, and mucosal protectants such as sucralfate are effective in promoting healing [4]. We report a case of self-expandable metal stent (SEMS) use for the treatment of recurrent hematemesis in a patient with late (chronic) radiation esophagitis refractory to medical therapy. Additionally, we discuss the management of radiation esophagitis and consideration for SEMS placement to promote mucosal healing by luminal exclusion.

1.1. Case. A 59-year-old Caucasian male with a history of stage IVA squamous cell carcinoma of the left mandible by TNM classification [5] presented with 1 day of hematemesis. His malignancy was diagnosed 2 years prior and treated by surgical resection followed by neoadjuvant chemoradiotherapy. His neoadjuvant regimen constituted of cisplatin, a total of $7200 \mathrm{cGy}$ radiation dose $(200 \mathrm{cGy} /$ fraction with 36 fractions) to the left retromolar trigone and $6480 \mathrm{cGy}$ radiation dose $(180 \mathrm{cGy} /$ fraction with 36 fractions) to the right neck. Recurrence 8 months prior to presentation prompted repeat radiation therapy performed at an outside institution, and hence, the retreatment protocol is not known. He had received supplemental enteral nutrition through endoscopic percutaneous gastrostomy tube for 2 years. He denied worsening dysphagia or odynophagia. Routine laboratory tests demonstrated a hemoglobin level of $9.7 \mathrm{~g} / \mathrm{dl}$ (laboratory normal: $13.5-17 \mathrm{~g} / \mathrm{dl}$ ). 


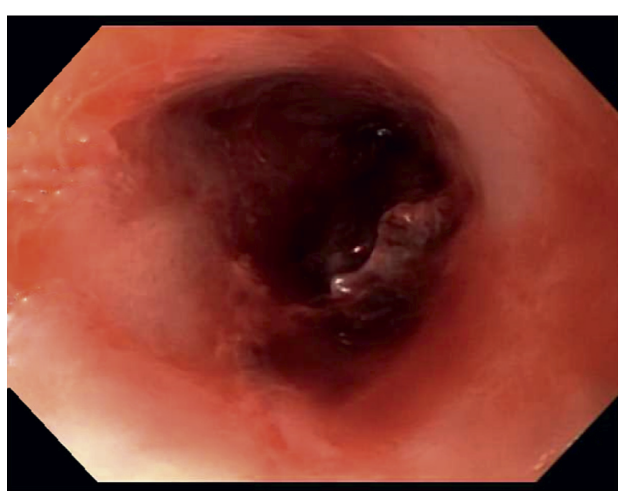

(a)

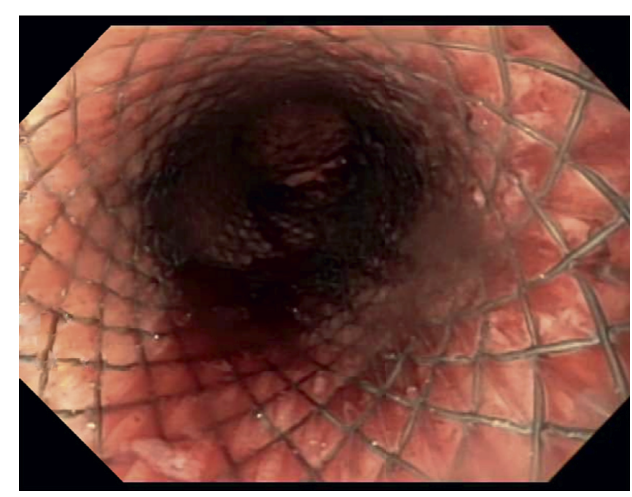

(b)

FIgURE 1: (a) Endoscopic appearance of distal esophagus demonstrating esophageal ulcer with active oozing (blue arrow). (b) Endoscopic appearance of distal esophagus following fully covered self-expanding metal stent (SEMS) (WallFlex ${ }^{\mathrm{TM}}$ Esophageal Stents, Boston Scientific, Marlborough, MA) deployment.

Intravenous pantoprazole $40 \mathrm{mg}$ twice daily was started, and esophagogastroduodenoscopy (EGD) was performed revealing diffuse erosive esophagitis and mild gastritis with no ulceration. No further bleeding ensued, and the patient was discharged home on omeprazole $40 \mathrm{mg}$ twice daily. He presented 5 months later with hematemesis, and a repeat EGD demonstrated a cratered $10 \mathrm{~mm}$ oozing esophageal ulcer at $33 \mathrm{~cm}$ from the incisors in a background of severe radiation esophagitis in the entire esophagus (grade 3 by Kuwahata's score [6]) (Figure 1(a)). Given the location of the lesion, active bleeding, and the lack of improvement with outpatient PPI therapy, a fully covered $23 \times 105 \mathrm{~mm}$ SEMS (WallFlex $^{\mathrm{TM}}$ Esophageal Stents, Boston Scientific, Marlborough, MA) was deployed to achieve hemostasis (Figure 1(b)). The patient was discharged only to return in 2 months with recurrent hematemesis. An abdominal radiograph demonstrated stent migration into the stomach (Figure 2(a)), and a repeat EGD was performed with stent removal. The cratered ulcer had completely healed at that time, but a background of diffuse severe esophagitis persisted (Figure 2(b)). Another identical stent was deployed. No further bleeding occurred, and he was discharged home with plans for replacement in 4 weeks.

Given difficulties accessing the hospital during the novel coronavirus (COVID-19) pandemic, he did not present for stent replacement and presented 5 months later with abdominal pain and was found to have recurrent stent migration. EGD demonstrated residual diffuse circumferential esophagitis, and the stent was removed. He continues to take omeprazole $40 \mathrm{mg}$ twice daily. Liquid sucralfate $1 \mathrm{~g}$ four times daily was added to his regimen. He had no bleeding recurrence on follow-up 5 months later.

\section{Discussion}

Achieving tumor control via radiotherapy without damage to adjacent tissues is difficult. Acute radiation esophagitis occurs in patients undergoing treatment for head and neck cancers. It occurs 2-3 weeks after the first treatment and can occur as late as 6 months to 1 year [7]. Patients often present with dysphagia, odynophagia, and substernal pain [7]. Esophageal stricture is a late complication that occurs 6-8 months after the first treatment dose and is a result of continual mucosal inflammation and fibrosis [7]. In addition, life-threatening complications such as perforation and fistulas can occur rendering early treatment of radiation esophagitis crucial [7].

Current mainstay prevention and treatment of radiation esophagitis includes diet modification to soft and bland diets [4]. Proton pump inhibitors (PPI), histamine blockers, antacids, and sucralfate are also used. However, use of these agents in patients with acute radiation esophagitis has yielded conflicting results. Sur et al. showed resolution of dysphagia and odynophagia along with improved ulcer healing time in patients receiving sucralfate compared with ones receiving antacids within 7 days of treatment [8], while another study showed no difference compared with placebo [9]. The use of free-radical neutralizing agents like amifostine may help relieve symptoms such as dysphagia. A prospective study demonstrated reduced esophagitis rates with use of prophylactic agents like glutamine [4].

The use of SEMS in radiation esophagitis is usually preserved for the treatment of symptomatic fibrotic benign strictures when dilations fail to relieve symptoms and/or luminal narrowing $[10,11]$. Studies evaluating the role of SEMS for esophageal ulceration with or without associated bleeding in radiation esophagitis are lacking. SEMS has been utilized in the control of esophageal variceal hemorrhage and is thought to control bleeding by a combination of tamponade and luminal exclusion promoting mucosal healing with success rates of $93.9 \%$ in a meta-analysis and a rebleeding rate of $13.2 \%$ [11-13]. In the reported case, a similar mechanism likely contributed to the resolution of the ulcer in 3 months following the first stent placement.

Stent migration remains a major concern with esophageal stenting and occurred twice in the reported case. In patients receiving SEMS for malignant dysphagia, stent migrations have been reported in $15.4 \%$ of patients, with higher risks in patients receiving fully covered SEMS, at $25.3 \%$ [14]. Endoscopic suturing or stent clipping to the 


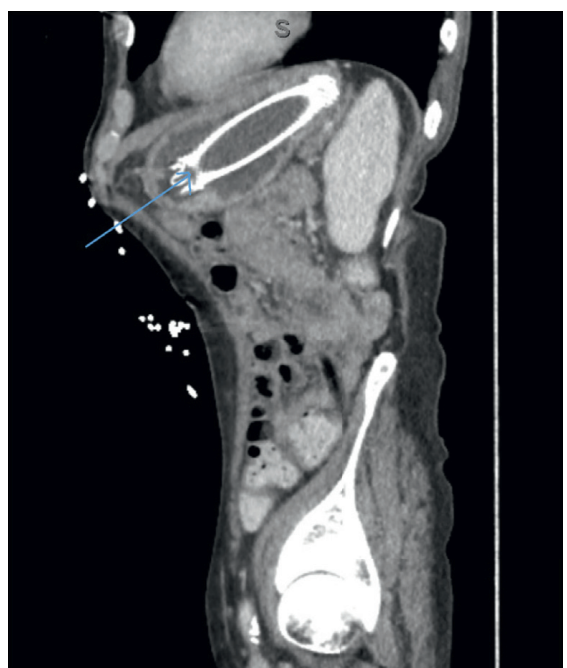

(a)

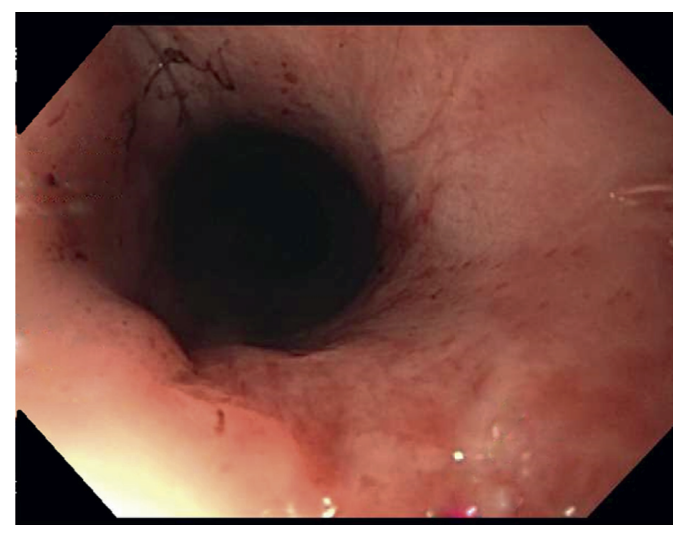

(b)

FIGURE 2: (a) Computed tomography (CT) of the abdomen demonstrating migration of self-expanding metal stent (SEMS) into the stomach (blue arrow). (b) Endoscopic appearance of distal esophagus demonstrating ulcer healing but with residual diffuse radiation esophagitis.

TABLE 1: Methodological appraisal of the reported case using the Murad tool [18] for risk of bias assessment of case reports and case series.

\begin{tabular}{lllllll}
\hline & Q1 $^{\text {i }}$ & Q2 $^{\text {ii }}$ & Q3 $^{\text {iii }}$ & Q4 $^{\text {iv }}$ & Q5 $^{\mathrm{v}}$ & Q6 $^{\text {vi }}$ \\
\hline Gill et al. & Yes & Yes & Yes & Yes & Yes & Yes \\
\hline
\end{tabular}

$\mathrm{i}$, does the patient represent the whole experience of the investigator? ii, was the diagnosis adequately ascertained? iii, was the outcome adequately ascertained? iv, were other alternative causes that may explain the observation ruled out? v, was follow-up long enough for outcomes to occur? vi, is the case(s) described with sufficient details to allow other investigators to replicate the research or to allow practitioners maker inferences related to their own practice?

esophageal wall are options that could be utilized to reduce risks of stent migration and in retrospect should have probably been utilized in this case $[15,16]$. Studies where SEMS were utilized for controlling variceal bleeding stent migration occurred in $21.6 \%$ in a meta-analysis [13] and in $38 \%$ in another case series [12]. The need for repeat endoscopy and stent removal adds to the overall morbidity and cost of utilizing SEMS in this setting. Concerns regarding tissue embedding following SEMS placement are uncommon in fully covered stents such as the one used [11]. Another important consideration is the risk of stent erosion particularly at the area of ulceration and formation of a tracheoesophageal or bronchoesophageal fistula requiring possible surgical repair [17]. In the reported case, we utilized a fully covered SEMS to promote mucosal healing, and tamponade bleeding in a patient with late (chronic) radiation esophagitis refractory to medical therapy. Migration of the SEMS into the stomach likely resulted in the rebleeding of the exposed friable mucosa leading to recurrent hematemesis. However, ulcer healing was observed, and no interval bleeding had occurred until stent migration ensued. Therapeutic measures for radiation esophagitis are limited, and untreated esophagitis leads to ulceration, fistula formation, perforations, and structure formation [4]. We postulate the possible use of SEMS as salvage therapy when conservative measures fail in treatment of bleeding secondary to late radiation esophagitis. A methodological appraisal of our case report using the Murad tool [18] is summarized in Table 1.

\section{Consent}

Informed consent was obtained from the patient.

\section{Conflicts of Interest}

The authors declare that they have no conflicts of interest.

\section{Authors' Contributions}

All the authors were involved with manuscript preparation and draft revisions and have reviewed and approved the manuscript for submission. I. G. and B. A. have performed a review on the literature on similar reported cases. G. H. has mentored and supervised the manuscript in its entirety.

\section{References}

[1] L. R. Coia, R. J. Myerson, and J. E. Tepper, "Late effects of radiation therapy on the gastrointestinal tract," International Journal of Radiation Oncology*Biology*Physics, vol. 31, no. 5, pp. 1213-1236, 1995.

[2] J. P. Desai and F. Moustarah, "Esophageal stricture," Stat Pearls, Treasure Island, FL, USA, 2020.

[3] D. Murro and S. Jakate, "Radiation esophagitis," Archives of Pathology \& Laboratory Medicine, vol. 139, no. 6, pp. 827-830, 2015.

[4] Z. Nesheiwat and S. S. Bhimji, Esophagitis, Radiation, StatPearls Publishing, Treasure Island, FL, USA, 2018, https:// www.ncbi.nlm.nih.gov/books/NBK499920/.

[5] S. H. Huang and B. O'Sullivan, "Overview of the 8th edition TNM classification for head and neck cancer," Current Treatment Options in Oncology, vol. 18, no. 7, 2017. 
[6] S. Hirota, K. Tsujino, Y. Hishikawa et al., "Endoscopic findings of radiation esophagitis in concurrent chemoradiotherapy for intrathoracic malignancies," Radiotherapy and Oncology, vol. 58, no. 3, pp. 273-278, 2001.

[7] S. Baker and A. Fairchild, "Radiation-induced esophagitis in lung cancer," Lung Cancer: Targets and Therapy, vol. 7, pp. 119-127, 2016.

[8] R. K. Sur, R. Kochhar, and D. P. Singh, "Oral sucralfate in acute radiation oesophagitis," Acta Oncologica, vol. 33, no. 1, pp. 61-63, 1994.

[9] W. L. Mc Ginnis, C. L. Loprinzi, S. J. Buskirk et al., "Placebocontrolled trial of sucralfate for inhibiting radiation-induced esophagitis," Journal of Clinical Oncology, vol. 15, no. 3, pp. 1239-1243, 1997.

[10] P. G. A. Van Boeckel and P. D. Siersema, "Refractory esophageal strictures: what to do when dilation fails," Current Treatment Options in Gastroenterology, vol. 13, no. 1, pp. 47-58, 2015.

[11] V. Kumbhari, P. Saxena, and M. A. Khashab, "Self-expandable metallic stents for bleeding esophageal varices," Saudi Journal of Gastroenterology, vol. 19, no. 4, pp. 141-143, 2013.

[12] M. S. Zakaria, I. M. Hamza, M. A. Mohey, and R. G. Hubamnn, "The first Egyptian experience using new selfexpandable metal stents in acute esophageal variceal bleeding: pilot study," Saudi Journal of Gastroenterology, vol. 19, no. 4, pp. 177-181, 2013.

[13] X.-D. Shao, X.-S. Qi, and X.-Z. Guo, "Esophageal stent for refractory variceal bleeding: a systemic review and metaanalysis," BioMed Research International, vol. 2016, p. 1, 2016.

[14] K. K. Das, S. Hasak, S. Elhanafi et al., "Performance and predictors of migration of partially and fully covered esophageal self-expanding metal stents for malignant dysphagia," Clinical Gastroenterology and Hepatology, vol. S1542-3565, 2020.

[15] K. Watanabe, T. Hikichi, J. Nakamura et al., "Feasibility of esophageal stent fixation with an over-the-scope-clip for malignant esophageal strictures to prevent migration," Endoscopy International Open, vol. 05, no. 11, pp. E1044-E1049, 2017.

[16] G. Vanbiervliet, J. Filippi, B. S. Karimdjee et al., "The role of clips in preventing migration of fully covered metallic esophageal stents: a pilot comparative study," Surgical Endoscopy, vol. 26, no. 1, pp. 53-59, 2012.

[17] K. R. Shen, M. S. Allen, S. D. Cassivi et al., "Surgical management of acquired nonmalignant tracheoesophageal and bronchoesophageal fistulae," The Annals of Thoracic Surgery, vol. 90, no. 3, pp. 914-919, 2010.

[18] M. H. Murad, S. Sultan, S. Haffar, and F. Bazerbachi, "Methodological quality and synthesis of case series and case reports," BMJ Evidence-Based Medicine, vol. 23, no. 2, pp. 60-63, 2018. 